\title{
Shock-Wave Compression and Joule-Thomson Expansion
}

\author{
Wm. G. Hoover and Carol G. Hoover \\ Ruby Valley Research Institute, Highway Contract 60, Box 601 Ruby Valley, Nevada 89833, USA \\ Karl P. Travis \\ Immobilisation Science Laboratory, Department of Materials Science and Engineering, University of Sheffield, \\ Mappin Street, Sheffield S1 3JD, United Kingdom \\ (Received 14 November 2013; published 10 April 2014)
}

\begin{abstract}
Structurally stable atomistic one-dimensional shock waves have long been simulated by injecting fresh cool particles and extracting old hot particles at opposite ends of a simulation box. The resulting shock profiles demonstrate tensor temperature, $T_{x x} \neq T_{y y}$ and Maxwell's delayed response, with stress lagging strain rate and heat flux lagging temperature gradient. Here this same geometry, supplemented by a shortranged external "plug" field, is used to simulate steady Joule-Kelvin throttling flow of hot dense fluid through a porous plug, producing a dilute and cooler product fluid.
\end{abstract}

DOI: 10.1103/PhysRevLett.112.144504

I. Stationary one-dimensional shock waves.-Shock waves are arguably farther from equilibrium than are any other readily available states of a nonequilibrium fluid [1-9]. In just a few collision times, or mean free paths, the shock transforms cold equilibrium fluid (or solid) into a hot compressed state [1-5]. Laboratory shock waves at a few terapascals can compress condensed matter as much as threefold, to densities and pressures far greater than those at the center of the Earth [6]. Because the shock transformation is a steady small-scale continuous process, converting kinetic energy to internal energy without any external heating, steady-state shock wave structures can be replicated with computer simulations [2-5,7-9]. The inset of Fig. 1 shows an interior snapshot of a typical (2D) simulation, with cold particles (initially arranged in a triangular lattice) entering at the left, and hot ones exiting to the right. The corresponding density profile snapshot using Lucy's weight function for the spatial averaging [7-11] is the smooth curve. The shock width can be estimated from the maximum slope. It is just a few atomic spacings. Steady-state profiles generated in this way are fully consistent with the transient profiles generated with [1] shrinking periodic boundaries or [2] head-on collisions of two similar blocks of cold material [5,7-9].

Both experiments and simulations show that initially sinusoidal shock fronts soon become planar. Steady shock waves are accurately one dimensional [3,5,7]. Accordingly, the mass, momentum, and energy fluxes ( in the $x$ direction, the propagation direction ) are all constant in the comoving coordinate frame of Fig. 1, the frame moving with the shock wave [1]:

Published by the American Physical Society under the terms of the Creative Commons Attribution 3.0 License. Further distribution of this work must maintain attribution to the author(s) and the published article's title, journal citation, and DOI.
PACS numbers: 47.40.-x, 47.11.Mn, 51.30.+i, 62.50.Ef

$$
\left\{\rho u ; P_{x x}+\rho u^{2} ;(\rho u)\left[e+\left(P_{x x} / \rho\right)+\left(u^{2} / 2\right)\right]+Q_{x}\right\}
$$

all three fluxes constant.

Here, $\rho(x)$ and $u(x)$ are the mass density and the flow velocity, $P_{x x}(x)$ is the pressure-tensor component in the propagation direction. $e(x)$ is the internal energy per unit mass, and $Q_{x}$ is the heat flux vector, measuring the conductive flow of heat in the comoving frame.

The cold entrance velocity is $+u_{s}$ (the "shock velocity") and the hot exit velocity is $+\left(u_{s}-u_{p}\right)$ (where $u_{p}$ is the "particle velocity") in the hot fluid. Away from the shock front the cold and hot pressure and energy have their thermodynamic equilibrium values:

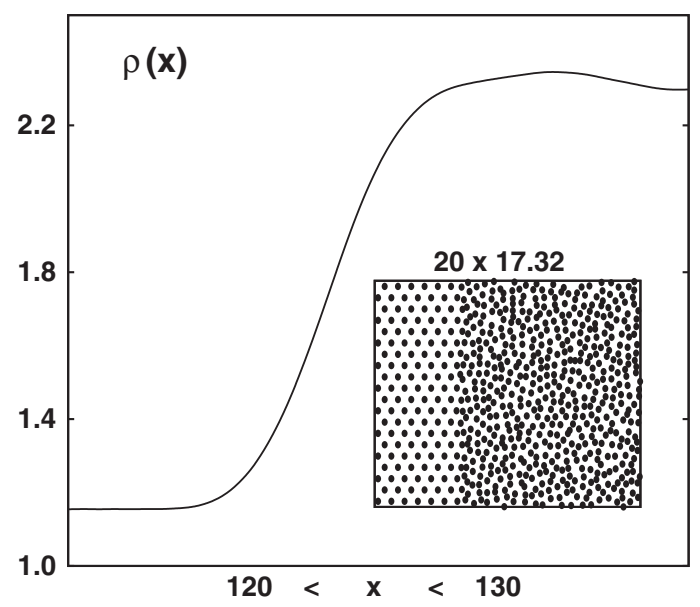

FIG. 1. Density profile and snapshot from a typical 1D shock wave simulation with a steeply repulsive pair potential in two space dimensions. Simulation cell dimensions $10 \sqrt{3} \times 250$. The spatially averaged density profile was obtained using Lucy's weight function. The snapshot shows a small section of length 20 near the shock front. 


$$
P_{x x}(x) \longrightarrow P_{\mathrm{eq}} ; \quad e(x) \longrightarrow e_{\mathrm{eq}} .
$$

Eliminating $u_{s}$ and $u_{p}$ from the three constant-flux equations gives the "Hugoniot equation" or "shock adiabat," $\Delta e=P \Delta v$, where $P$ is the mean pressure, $\left[P_{\text {cold }}+P_{\text {hot }}\right] / 2$, and $\Delta v$ is the overall change in volume per unit mass, $(1 / \rho)_{\text {cold }}-(1 / \rho)_{\text {hot }}$. Though there is no external heating there is heat flow within the shock wave structure. For weak shock waves it is given by Fourier's law, $Q_{x}=$ $-\kappa(d T / d x)$.

The limiting values of the energy flux divided by the mass flux far from the shock wave are equal:

$\left[e+(P / \rho)+\left(u_{s}^{2} / 2\right)\right]_{\mathrm{cold}}=\left[e+(P / \rho)+(1 / 2)\left(u_{s}-u_{p}\right)^{2}\right]_{\text {hot }}$.

In shock waves the inflow is supersonic so that the kinetic energy cannot be ignored. Choosing the initial thermodynamic state along with the particle velocity determines the shock velocity as well as the pressure and energy of the resulting "hot" state.

Joule-Thomson "throttling" flows.-In the 1850s Joule and Thomson (who became Lord Kelvin in 1892) collaborated on the design and analysis of experiments seeking to quantify the "mechanical equivalent of heat." The "JouleThomson," or "Joule-Kelvin," experiment enforced the throttling of a high-pressure gas through a porous plug [12]. A detailed description of the evolution of these experiments can be found in Ref. [14]. Within the plug the inlet pressure is reduced to the smaller outlet pressure. As the flow rate approaches zero the experiment becomes isenthalpic, where the enthalpy is $E+P V$. Because there is no external heat flow the work added at the hot highpressure side less that extracted on the cold low-pressure side is the energy change:

$$
[e+(P / \rho)]_{\text {high } \mathrm{P}} \rightrightarrows[e+(P / \rho)]_{\text {low } \mathrm{P}}[\text { Joule-Thomson }] \text {. }
$$

By contrast to the supersonic shock wave experiment, kinetic energy is negligible in the typical laboratory JouleKelvin experiment. The conductive heat flux (a maximum at the shock front) is likewise invisible in the throttling experiment, concealed by the irreversible details of the porous plug. Otherwise, the geometry and the thermodynamics and the constancy of the fluxes look identical to the usual one-dimensional shock wave analyses. In both experiment types there is necessarily a positive entropy change within the flow, as is required by the second law of thermodynamics.

Joule-Thomson simulations. - The structural similarity of shock wave compression and Joule-Kelvin expansion experiments suggests the possibility of simulating JouleKelvin flows with molecular dynamics. Here we validate and illustrate that idea for a 2D system. Our model must incorporate a computational "porous plug" to slow compressed input fluid. Pores, holes, and confining passageways come to mind. But a little reflection suggests a simpler approach-erecting a smooth potential-energy barrier perpendicular to the flow. This approach is successful. Apart from the entrance and exit boundaries, the motion is entirely conservative and Newtonian. An initial configuration, twice as dense on the left as on the right, is created from a square lattice. Fresh columns of particles are continually fed into the simulation from the left and are removed once they reach the rightmost edge of the simulation box (conveyor-belt boundaries). The entrance internal energy can be controlled by adding $y$ displacements (to control potential energy) and/or Maxwellian velocities $\left(v_{x}-u, v_{y}\right)$ to particles as they enter (controls the kinetic energy). In our simulation, the entrance velocity $u$ was 0.5 , exit velocity was 1.0 , and initial temperature was 0.05 .

Near the potential plug barrier an anisotropic far-fromequilibrium state results. The fluid is first slowed and then accelerated normal to the barrier, with the result that the pressure and temperature are briefly anisotropic with $P_{x x}>$ $P_{y y}$ and $T_{x x}>T_{y y}$. The details of the equilibration involve the same Maxwellian [13] time delays seen in shock waves.

Figure 2 shows a typical Joule-Thomson steady-state particle snapshot, with similar pair and barrier potentials chosen to minimize integration errors using fourth-order Runge-Kutta molecular dynamics with a time step $d t=0.01$ :

$$
\begin{aligned}
\phi_{\text {pair }}(r<1) & =\left[1-r^{2}\right]^{4} ; \\
\phi_{\text {barrier }}(-1<x<+1) & =(1 / 4)\left[1-x^{2}\right]^{4} .
\end{aligned}
$$

Although such a potential was perfectly satisfactory for the shock wave simulations of twofold compression it suggests the possibility of poor behavior at high density, where the force is a decreasing function of compression. Accordingly, we compared results with a modified pair potential for which the force remains constant, with its maximum value $F_{\max }$ at separations less than $r_{\max }=\sqrt{(1 / 7)}=0.377964473$ :



FIG. 2. A Joule-Thomson snapshot. The motion is left-to-right with cooled fluid exiting at the right boundary. The density snapshot uses Lucy's weight function, $(5 / 12)(1+|x|)[1-(|x| / 3)]^{3}$. 


$$
\begin{aligned}
& \phi_{\max }\left(r<r_{\max }\right)=(6 / 7)^{4}+F_{\max }\left(r-r_{\max }\right) ; \\
& F_{\max }\left(r<r_{\max }\right)=8(6 / 7)^{3} \sqrt{(1 / 7)} .
\end{aligned}
$$

Joule-Thomson profiles including this $\phi_{\text {pair }}$ precaution were not significantly changed from those with the unmodified potential.

Corresponding time-averaged density and velocity profiles are shown in Fig. 3, along with the (necessarily constant) mass flux, $\rho u$. Just as in our shock work the onedimensional grid-profile averages were all computed using Lucy's one-dimensional smooth-particle weight function [7-11], with $h=3$ :

$$
\begin{aligned}
\left\langle f\left(x_{g}\right)\right\rangle & =\sum_{x_{j}>x_{g}-h}^{x_{j}<x_{g}+h} f_{j} w_{g j} ; \\
w_{g j} & =(5 / 12)\left[1+\left|x_{g j}\right|\right]\left[1-\left(\left|x_{g j}\right| / 3\right)\right]^{3} \\
& \rightarrow \int_{-3}^{+3} w(|x|) d x \equiv 1 .
\end{aligned}
$$

With an input speed of 0.5 , which quickly accelerates to 0.62 , the velocity speeds up to 1.25 on passing through the plug potential. Straightforward Runge-Kutta simulation converges relatively simply and quickly to a flow satisfying slight modifications of the conservation relations which hold for shock wave simulations.

The longitudinal momentum flux drops at the barrier because the barrier force removes momentum. The overall flux drop exactly matches $\left(F_{\text {barrier }} / L_{y}\right)$, with

$$
\left[P+\rho u^{2}\right]_{\text {left }}=\left[P+\rho u^{2}\right]_{\text {right }}-\left(F / L_{y}\right)[F \text { negative }] .
$$

Mass, momentum, and energy fluxes are shown in Fig. 3.

The energy flux is particularly interesting. Adding the contributions of pair interactions $\left(\left(\dot{x}_{i}+\dot{x}_{j}\right) / 2\right) x_{i j} F_{i j}^{x}$ to the "convective flux" gives perfect agreement between the entrance and exit flows. These contributions can be divided equally between particles $i$ and $j$. Alternatively, they can be velocity weighted: $\left(\dot{x}_{i} / 2\right) x_{i j} F_{i j}^{x}$ for $i$ and $\left(\dot{x}_{j} / 2\right) x_{i j} F_{i j}^{x}$ for $j$. The effect of this choice on the energy flux is insignificant, of order 0.001 . In shock waves the total pressure-tensor component $P_{x x}$ includes the $\rho k T_{x x}$, which is absent in our Joule-Thomson flux. The derivations for these two slightly different expressions for the energy flux are both familiar textbook fare [15]. The reason for the difference is interesting. The $x$ component of the purely kinetic part of the energy flux (excluding the contributions from $\phi$ and $F$ ) involves local sums cubic in the velocity components. In the equilibrium case the cubic sum can be expressed in terms of the stream velocity and the deviations from it, which can in turn be expressed in terms of temperature:

$$
\begin{aligned}
\left\langle\left(v_{x} / 2\right)\left(v_{x}^{2}+v_{y}^{2}\right)\right\rangle= & (1 / 2)\left\langle\left(u+\delta v_{x}\right)^{3}+u\left(\delta v_{y}\right)^{2}\right\rangle \\
= & (1 / 2) u^{3}+(3 / 2) u k T_{x x} \\
& +(1 / 2) u k T_{y y} .
\end{aligned}
$$

The resulting "extra" $\rho u k T_{x x}$ can, if desired, be combined with the potential part of $u P_{x x}$ so as to agree with the continuum energy-flux expression. Far from equilibrium this simplification does not hold and the full cubic kinetictheory sums must be evaluated.

In general, it is interesting to note that the hot and cold momentum fluxes don't match in the Joule-Kelvin experiment though they do in the shock wave. (If both the fluxes, energy and momentum, were to match, either the shock wave or the throttling experiment would violate the second law.) The reason for the flux drop at the barrier is the latter's contribution to the momentum flux, by exerting a nonzero compressive force on the hot fluid. In our demonstration problem the fluid is cooled substantially, in keeping with the familiar commercial mechanism using throttling as a model refrigerator. Like shock waves, the present highspeed Joule-Thomson flows are contained by equilibrium thermodynamic boundaries. A series of Joule-Thomson states can be generated by using several plug barriers rather than just one. Accordingly, we believe that they will, like shock waves, provide a useful source of
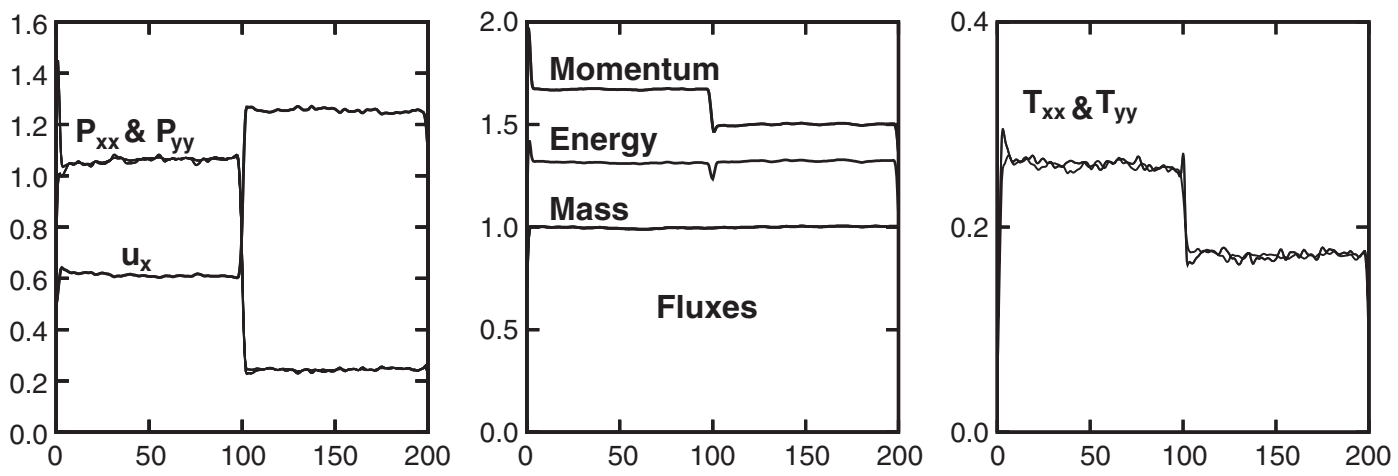

FIG. 3. Time-averaged pressure tensor and velocity (left); time-averaged mass, momentum, and energy fluxes (centre); tensor temperature (right). The system dimensions are $200 \times 40$. The mass flux of unity is imposed by the rate at which fresh particles are inserted at the left boundary. 
computer-experimental constitutive information for flow states far from equilibrium and help in choosing the optimum weight function for correlating microscopic and macroscopic flow descriptions.

[1] L. D. Landau and E. M. Lifshitz, Fluid Mechanics (Pergamon, New York, 1959). Chapter IX is devoted to shock waves.

[2] R. E. Duff, W. H. Gust, E. B. Royce, M. Ross, A. C. Mitchell, R. N. Keeler, and W. G. Hoover, Proceedings of the 1967 Paris Conference (Gordon and Breach, New York, 1968), p. 397.

[3] V. Y. Klimenko and A. N. Dremin, Detonatsiya, Chernogolovka (Akademia Nauk, Moscow, 1978), p. 79.

[4] Wm. G. Hoover, Phys. Rev. Lett. 42, 1531 (1979).

[5] Brad Lee Holian, Wm. G. Hoover, Bill Moran, and Galen K. Straub, Phys. Rev. A 22, 2798 (1980).

[6] C. E. Ragan, III, Phys. Rev. A 29, 1391 (1984).
[7] Wm. G. Hoover and C. G. Hoover, Phys. Rev. E 80, 011128 (2009).

[8] Wm. G. Hoover, C. G. Hoover, and F. J. Uribe, arXiv:1005.1525.

[9] Wm. G. Hoover and C. G. Hoover, Computer Simulation, Time Reversibility, Algorithms, and Chaos (World Scientific, Singapore, 2012). Chapter 6 is devoted to shock waves.

[10] L. B. Lucy, Astron. J. 82, 1013 (1977).

[11] Wm. G. Hoover, Smooth Particle Applied Mechanics-The State of the Art (World Scientific, Singapore, 2006).

[12] W. Thomson and J. P. Joule, Phil. Trans. R. Soc. London 143, 357 (1853).

[13] J. C. Maxwell, Phil. Trans. R. Soc. London 157, 49 (1867).

[14] J. S. Rowlinson, Notes and Records Roy. Soc. 64, 43 (2010).

[15] Wm. G. Hoover, Molecular Dynamics (Springer-Verlag, Berlin, 1986); available without charge at http:// williamhoover.info. See Sections II.B and II.C. 